\title{
A new simulation environment to model spontaneous and evoked activity of large-scale neuronal networks coupled to micro-electrode arrays
}

\author{
Marcello Mulas*, Sergio Martinoia and Paolo Massobrio
}

Address: Department of Biophysical and Electronic Engineering (DIBE), University of Genova, Genova, Italy

Email: Marcello Mulas* - marcello.mulas@unige.it

* Corresponding author

from Eighteenth Annual Computational Neuroscience Meeting: CNS*2009

Berlin, Germany. 18-23 July 2009

Published: 13 July 2009

BMC Neuroscience 2009, I0(Suppl I):P348 doi:I0.I |86/I47|-2202-I0-SI-P348

This abstract is available from: http://www.biomedcentral.com/I47I-2202/I0/SI/P348

(c) 2009 Mulas et al; licensee BioMed Central Ltd.

\section{Introduction}

The use of neuronal cultures coupled to Micro-Electrode Array (MEA) is becoming a widely used and recognized experimental model for studying basic properties of information processing in neuronal systems [1]. However, the electrophysiological activity of such large-scale neuronal networks is recorded only by tens/hundreds microelectrodes. This undersampling results in a lack of information. Thus the development of a new simulation environment, able to reproduce the electrophysiological behavior typically found in these preparations, offers promise in better understanding the actual dynamics. In this work, we present the main features of our software, showing the simulation results of the spontaneous and evoked activity of a highly-connected network.

\section{Methods}

The simulation environment is developed in $\mathrm{C \#}$, the last generation object-oriented language by Microsoft. The choice of this language is a good tradeoff between our needs of high performance and speed in algorithm implementation. The software was designed to support easily further neural network models, so that it will be possible to use the more suitable model according to the desired physiological accuracy and computational efficiency. The user is able to define the initial topology of a network (e.g., random, small-world, scale-free, etc.) by changing several parameters that modify the probability to have a link between two neurons. It is also possible to define dif- ferent stimulation points in the network in order to simulate the evoked activity. Finally, the user can also take advantage of a detailed visual representation of the simulated electrophysiological activity (raster plots, membrane potential levels, etc.) and of the connectivity of the network (links, false color map of the synaptic weights, etc.). Figures 1a-d show some examples of graphical representations.

\section{Results}

We simulated a neuronal network model made up of 1024 point neurons, each modeled according to the Izhikevich equations [2]. In particular, we considered two different types of neuron to model excitatory and inhibitory populations of neurons, respectively: the former belongs to the family of regular spiking neurons while the latter to the family of fast spiking neurons. To preserve
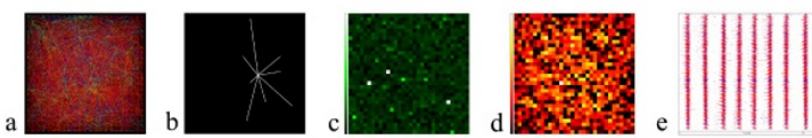

Figure I

a) Network links, b) detail of the links of a single neuron, c) neuron membrane potentials, d) sum of output synaptic weights, e) raster plot of $60 \mathrm{~s}$ of simulated electrophysiological activity. 
some characteristics of the structure of in vitro cortical neurons, we set the ratio between excitatory and inhibitory neurons to $4: 1$. [1] We first simulated the spontaneous activity observing the typical behavior found experimentally: a mix of spiking and bursting activity (Figure 1e). In addition, we also simulated the evoked activity elicited by a low frequency electrical stimulation $(0.2 \mathrm{~Hz})$, finding that the high network connectivity produces a synchronized and distributed response that involves almost all the neurons.

\section{Conclusion}

In this work we presented a new simulation environment, designed as a support tool for electrophysiological experiments with neuronal cultures coupled to MEA. The main strengths of this simulator are the efficient visualization of the neural activity and the possibility to define different connectivity rules and stimulation points.

Our preliminary simulations show that we are able to reproduce both the spontaneous and evoked activity.

\section{References}

I. Marom S, Shahaf G: Development, learning and memory in large random networks of cortical neurons: lessons beyond anatomy. Quarterly Reviews of Biophysics 2002, 35:63-87.

2. Izhikevich EM: Simple model of spiking neurons. IEEE Transactions on Neural Networks 2003, 6:1569-1572.
Publish with Bio Med Central and every scientist can read your work free of charge

"BioMed Central will be the most significant development for disseminating the results of biomedical research in our lifetime. " Sir Paul Nurse, Cancer Research UK

Your research papers will be:

- available free of charge to the entire biomedical community

- peer reviewed and published immediately upon acceptance

- cited in PubMed and archived on PubMed Central

- yours - you keep the copyright

Submit your manuscript here:

http://www.biomedcentral.com/info/publishing_adv.asp 